\title{
Evaluation of rotational resistance, and rotational and vertical discrepancy of three different elastomeric impression materials with open tray implant level impressions on a special model
}

\author{
Ambedkar Elumalai, Saravanakumar Mariappan, ChitraShankar Krishnan, \\ Hariharan Ramasubramanian, Jayakrishnakumar Sampathkumar, Hariharan Ramakrishnan, \\ Azhagarasan Nagarasampatti Sivaprakasam, Vallabh Mahadevan
}

Department of Prosthodontics \& Implantology, Ragas Dental College \& Hospital, The TN Dr MGR Medical University, Chennai, India

Purpose: This study was conducted to comparatively evaluate of the rigidity and accuracy of three different elastomeric impression materials with an open tray implant level impression technique.

Materials and Methods: In Part I of the study, a total of thirty open tray implant level impressions were made with vinylpolysiloxane $(n=10)$, polyether $(n=10)$, and vinylsiloxanether $(n=10)$ impression materials. Implant replicas were connected to the impression coping using a digital screw torque checker. The peak torque values were recorded, indicating the maximum rigidity of each group. The mean values were obtained and statistically analyzed using one-way ANOVA and the post-hoc Tukey's Honestly Significant Difference (HSD) test. Similarly, in Part Il of the study, a total of thirty impressions were made with vinylpolysiloxane $(n=10)$, polyether $(n=10)$, and vinylsiloxanether $(\mathrm{n}=10)$ impression materials. The implant replicas were connected to the impression coping with a $5 \mathrm{~N} \cdot \mathrm{cm}$ torque for the vinylpolysiloxane and polyether groups. A gentle hand tightening was given to the vinylsiloxanether group. The master casts were then poured. Subsequently, linear angle and vertical distance were measured using a coordinate measuring machine. The mean values were obtained and statistically analyzed using one-way ANOVA, post-hoc Tukey's HSD, and an independent 't' test.

Results: Vinylpolysiloxane showed the maximum rotational resistance followed by polyether and the least by vinylsiloxanether, and the differences were statistically significant $(\mathrm{P}<0.05)$. Polyether showed the least rotational discrepancy compared to vinyl polysiloxane and vinylsiloxanether, and the variations were statistically significant $(P<0.05)$. Vinylpolysiloxane showed the least vertical discrepancy compared to vinylsiloxanether and polyether without any statistical significance $(P>0.05)$.

Conclusions: Among the elastomeric impression materials used in this study, vinylpolysiloxane showed the maximum rotational resistance, polyether showed the least rotational discrepancy, and the vertical discrepancy between these three materials was not significant. (JOURNAL OF DENTAL IMPLANT RESEARCH 2021;40(3):66-75)

Key Words: Dental impression material, Dental impression technique, Silicones, Vinylpolysiloxane, Dental implant

\section{INTRODUCTION}

An endosteal implant is an alloplastic material surgically inserted into a residual bony ridge primarily as a prosthodontic foundation ${ }^{1)}$.

The fit of a restoration can be considered "passive" if it does not create any static loads within the prosthetic system or in the surrounding bone tissue. Passive fit is

Received May 14, 2021, Revised July 7, 2021, Accepted July 28, 2021.

(c) Journal of Dental Implant Research.

This is an open access article distributed under the terms of the Creative Commons Attribution Non-Commercial License

(http://creativecommons.org/licenses/by-nc/4.0) which permits unrestricted non-commercial use, distribution, and reproduction in any medium, provided the original work is properly cited.

Correspondence to: Hariharan Ramakrishnan, https://orcid.org/0000-0003-4466-5744

Department of Prosthodontics \& Implantology, Ragas Dental College \&Hospital, Uthandi, Chennai 600119, India. Tel: +91-44-24530006, Fax: +91-44-52123995, E-mail: abcv2005@yahoo.com 
affected by various factors such as implant position, impression materials, impression technique, and expansion of gypsum casts, investing and casting procedures for the fabrication of metal framework ${ }^{2-5}$. In case of misfit between implant and abutment as well as between abutment and prosthesis, compressive and traction loads could be directed to the restoration, resulting in loosening of the prosthesis and abutment screws, fracture of the restoration, bone microfractures surrounding the implants, and even fracture of the implant body ${ }^{2,6-8)}$. Such a misfit will also lead to problems with the articulation of the working cast, axial contouring of interproximal contacts, open margins, lack of retention, and resistance to displacement. Marginal discrepancies caused by the misfit might enhance plaque accumulation, affecting soft and/or hard tissue around the implants ${ }^{9)}$. The accuracy of impression is influenced by various factors such as depth and angulations of implants, the position of implants, impression materials, impression technique, type of impression trays, different connection level (implant or abutment level), design of impression copings, splinting or non-splinting of impression copings, time delay for impression pouring ${ }^{11-13)}$. The indirect (closed tray) and direct (open tray) techniques have been advocated in the literature for implant/abutment level impressions to ensure the passive fit of the prosthesis ${ }^{4,14)}$.

Implant level indirect transfer for closed tray impression technique involves tapered impression copings which would be repositioned along with the replicas within the impression ${ }^{7,15,16)}$. The advantage of this technique is that the implant replicas are visually fastened to the impression copings and therefore ensuring its complete seating ${ }^{7}$. But on the other hand, the reseating of the coping in the elastic impression may not be accurate, which can reflect an error in the inter-implant relationship in the vertical axis. The instances of impression material being distorted or damaged are also possible while using the closed tray impression technique in multiple implant situations, especially if implants are not parallel to each other ${ }^{7,17)}$.

To overcome the deficiencies associated with the closed tray impression technique, the open tray implant level impression technique has been introduced for single as well as multiple implant situations. This open tray im- pression uses square copings and an open tray (a tray with an opening) allowing the head of the impression copings screw to be exposed. The advantage is that the coping remains within the impression and so there need not be any concern for replacing it into its respective space $^{4,7,18)}$. Also, the concern of angulated implants deforming the impression material upon removable of impression does not arise. There is no consensus found in the literature for the amount of torque to be applied while connecting the implant replica to the open tray impression ${ }^{6,11,19)}$. The blind fastening of the implant replica can result in rotation of the impression coping and thus causes the rotational discrepancy ${ }^{7,11,20)}$.

Splinting of open tray impression copings has been suggested by many authors to maintain the accurate inter-implant relationship when compared to that obtained with non-splinted impression copings ${ }^{5,11,18)}$. However splinting of the open tray impression coping is technically not possible with a single implant situation or randomly distributed single implant situations in a partially edentulous arch. Individual variations in hand-tightening torque might lead to rotation of impression copings within the impression $^{21)}$. Such an error is likely to influence the passive fit of the abutment/ prosthesis, contact points with the adjacent teeth, and occlusion. The rotational tendency of the open tray impression copings while connecting the implant replica is influenced by the rigidity of the impression material and the design of the impression coping $^{11,13,18)}$.

The impression material used for making open tray impressions should possess sufficient rigidity to prevent accidental rotation of the coping and exhibit minimal positional distortion. Various impression materials are recommended for making implant impressions which will include polyether and additional silicone ${ }^{5,18,22,23)}$. Polyether has been suggested for completely edentulous multiple-implant situations due to its excellent resistance to permanent deformation, favorable shore hardness, low strain under compression, and high tear resistance ${ }^{24)}$. The use of polyether impression material for a partially edentulous situation presents the difficulty of retrieving the set impressions intraorally because of its high rigidity ${ }^{25)}$. Addition silicone with its more favorable modulus of elasticity allows easy removal of the set impression in 

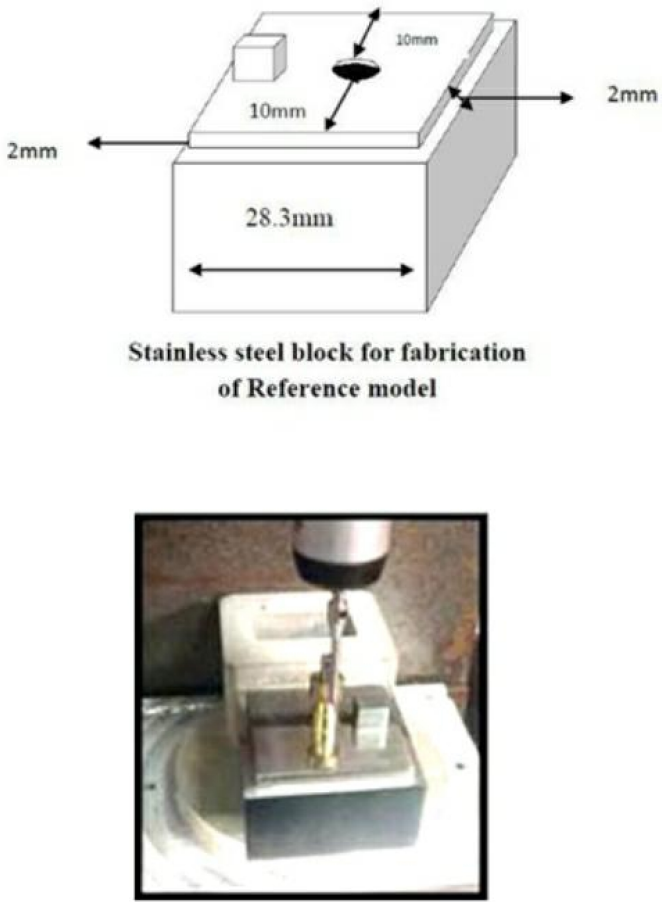

Fig. 1. Stainless steel block for fabrication of reference model and measurement of the linear angle on the reference model using a coordinate measuring device.

such situations ${ }^{24,25)}$. Hybrid elastomeric impression material vinylsiloxanether has been introduced to encompass the desirable properties of vinylpolysiloxane and polyether materials for making impressions. There is one clinical study reported in the literature regarding the use of vinylsiloxanether impression material in implant impressions $^{26)}$.

The rotational tendency of the impression coping in an open tray impression is influenced by the properties of the impression materials, design of the impression copings, splinting/non-splinting, coating of tray adhesive, and individual variability of hand tightening torque $^{6,11,13,21)}$.

\section{MATERIALS AND METHOD}

Cuboid stainless steel block was fabricated with the dimensions of $28.3 \mathrm{~mm} \times 28.3 \mathrm{~mm} \times 16 \mathrm{~mm}$. A cylindrical well was milled in the center of the stainless steel block with a diameter of $4.3 \mathrm{~mm}$ and a depth of $11 \mathrm{~mm}$. An elevated rectangular projection with dimensions of 4.3 $\mathrm{mm} \times 5 \mathrm{~mm} \times 4 \mathrm{~mm}$ was milled parallel to the cylindrical well during the fabrication of the metal block. A step fea-
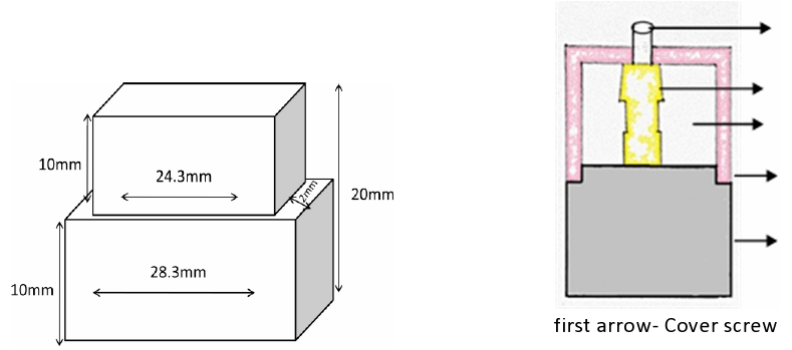

Second- Impression

coping

Third -Space for

impression

Fourth- Custom tray

Fifth- Ref model

Fig. 2. Stainless steel block for custom tray fabrication.

ture was milled on the periphery of the stainless steel block with a dimension of $2 \mathrm{~mm} \times 2 \mathrm{~mm}$ to aid in the orientation of the custom open tray (Fig. 1).

Implant replica of $4.3 \mathrm{~mm}$ diameter with the internal tri-channel connection was connected to its respective open tray impression coping. The cylindrical wall of the stainless steel block and axial surfaces of the implant replica were air abraded with $110 \mu \mathrm{m}$ alumina particles. The flat surfaces of the impression coping were aligned parallel to the flat surface of the rectangular projection using a linear height master. The Type I glass ionomer cement was mixed according to the manufacturer's instruction and applied to walls of the cylindrical well and the axial surface of the implant replica. The implant replica-impression coping assembly was cemented to the cylindrical well of the stainless steel block in such a way that the interface of the implant replica-impression coping assembly was kept level with the top plane of the stainless steel block. The flat surface of the open tray impression coping was aligned parallel to the flat surface of the rectangular projection of the stainless steel block using linear height master initially followed by-ordinate measuring machine before the cement set. Thus, a reference model was obtained.

Stainless steel block was fabricated with the dimensions of $28.3 \mathrm{~mm} \times 28.3 \mathrm{~mm} \times 20 \mathrm{~mm}$ thickness. A stepped design with the dimensions of $2 \mathrm{~mm} \times 10 \mathrm{~mm}$ was incorporated at the periphery. This device was used for the fabrication of a custom open tray with standardized 

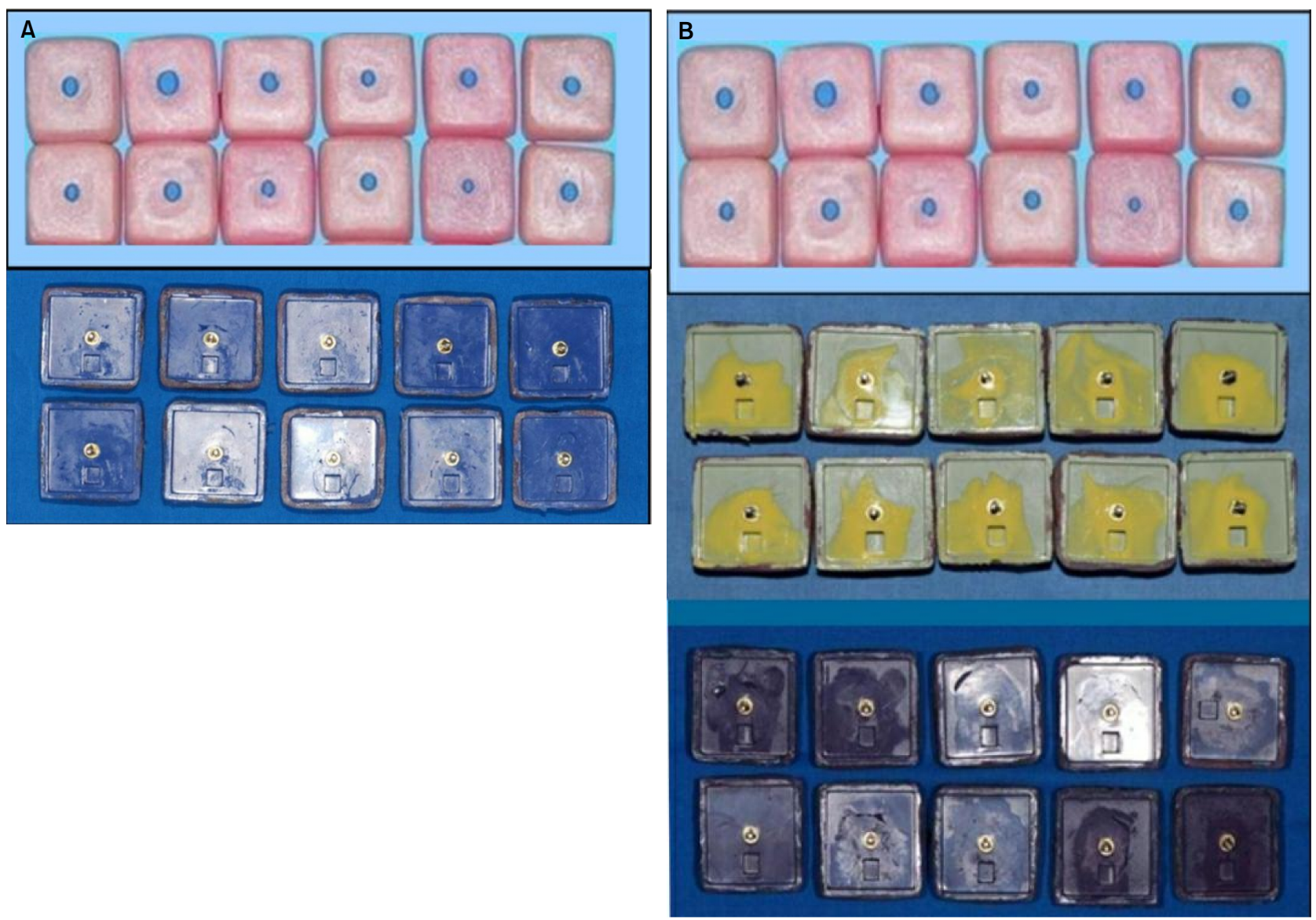

Fig. 3. (A) Custom trays to evaluate rigidity of the impression material (Part 1), Open tray implant level impressions with vinylsiloxanether impression material. (B) Custom trays to evaluate rotational and vertical discrepancy (Part 2), Open tray implant level impressions with vinylpolysiloxane impression material, Open tray implant level impressions with polyether impression material.

spacer thickness (Fig. 2).

A light polymerizing resin sheet of $2 \mathrm{~mm}$ thickness was adapted on the stainless steel block. Later, it was placed inside the light-curing unit for 6 minutes and light-cured. The tray was removed from the model and kept inside the curing unit for another 6 minutes. A round opening was made on the occlusal surface of the tray to gain access to the open tray impression coping screws. The finished tray was placed on the reference model to verify its proper orientation. In this manner, 60 custom trays were made for open tray implant level impressions. All the trays were left undisturbed for 24 hours, for the trays to become dimensionally stable before impression making.

A total of 60 custom trays were fabricated. Thirty custom trays were randomly selected and used for making open tray implant level impressions for evaluating the ri- gidity of the impression materials (Evaluation of rotational resistance, Part 1, comprising Group 1A, 1B, 1C representing VPS, Polyther, VSE) (Fig. 3A). The remaining thirty custom trays were used for making open tray implant level impressions for evaluating the accuracy of the impression materials (Evaluation of rotational discrepancy and vertical discrepancy, 11A, 11B, 11C) (Fig. 3B).

\section{Evaluation of rotational resistance}

Open tray implant level impression coping (Nobel Replace Internal tri-channel; REF 29500, Nobel Biocare $\mathrm{AB}$, Goteborg, Sweden) was connected to the implant replica of the reference model with the screwdriver. The custom tray was coated with a thin layer of vinylpolysiloxane tray adhesive (3M ESPE; Seinfeld, Germany) and allowed to dry for fifteen minutes as per the manu- 


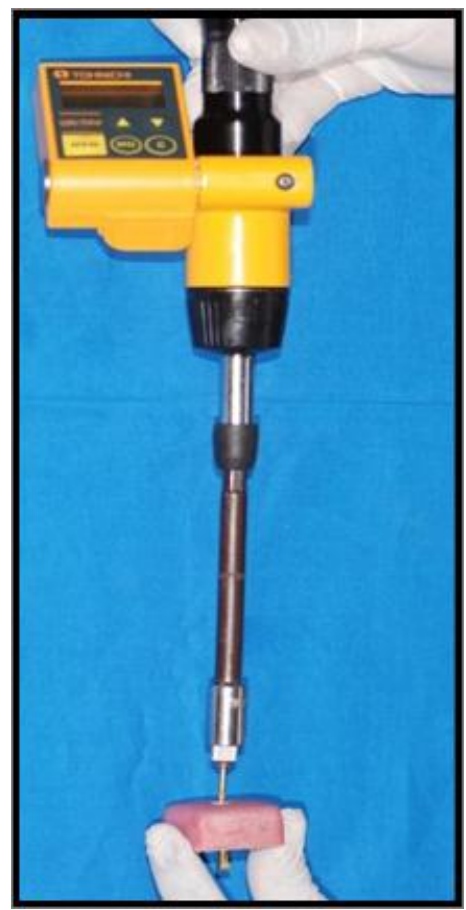

Fig. 4. Digital Screw Torque Checker evaluating the rotational resistance.

facturers' recommendation. Putty vinylpolysiloxane impression material (Express XT Penta putty; 3M ESPE, Seinfeld, Germany) was machined mixed (Pentamix 2, 3M ESPE, Seinfeld, Germany) and loaded into the custom tray keeping the tip immersed in the material all the time. The light body (Light body Express XT; 3M ESPE, Seinfeld, Germany) was syringed around the impression coping by another operator simultaneously. The tray was positioned on the reference model immediately. The impression was allowed to sit undisturbed for 3 minutes as per the manufacturers' After polymerization, the coping screw was unscrewed and the impression was retrieved from the reference model. A total of ten open tray implant level impressions were made using vinylpolysiloxane impression material and they are designated as Group IA. Using Pentamix technique for Polyether, and by following manufacturer recommendations ten samples were prepared and designated as Group 1B. group 1C consisted of ten samples of vinylsiloxane ether.

Implant replica of the Nobel Replace system was connected to the impression coping with a screwdriver. Gentle hand tightening was done to secure the implant replica to the impression coping. The screwdriver was fixed to the adapter of the digital screw torque checker. The adapter was connected to the digital screw torque checker. The ratchet change-over knob of the digital screw torque checker was adjusted to the 'L' position and the memory number was assigned in the digital screw torque checker to each impression before checking the rotational resistance. A digital screw torque checker was used to torque the coping screws by turning the plastic handle in a clockwise direction until the rotation of impression coping. As the coping screws were tightened, the increasing torque values were displayed on the digital screw torque checker. Once the peak torque values were achieved there was a decrease in the torque values, indicating that impression coping starts rotating within the impression. The peak torque value was alone displayed on the digital screw torque checker. The auto-memory function of this device stored the peak torque value obtained for that impression (Fig. 4). This torque value represented the rotational resistance offered by the impression material with the open tray impression coping.

\section{Evaluation of the rotational and vertical discrepancy}

The implant replica of the corresponding system was connected to the impression coping within the impression using a screwdriver. Gentle hand tightening was done to secure the implant replica to the open tray impression coping. A digital screw torque checker was used to torque the coping screw by turning the plastic handle in a clockwise direction until $5 \mathrm{~N} \cdot \mathrm{cm}$ torque was displayed as described in Part I. The same procedure was repeated for Group IIA (vinylpolysiloxane, $n=10$ ) and Group IIB (polyether, $\mathrm{n}=10$ ) impressions. A gentle hand tightening was alone given to secure implant replica to the impression copings for Group IIC (vinylsiloxanether, $n=10$ ) impressions. The above impressions were boxed and Type IV dental stone was mixed with water as per the manufacturer's recommendation of $100 \mathrm{~g}$ to $20 \mathrm{ml}$. The mixed dental stone was poured within the boxed impression using a vibrator. The cast was allowed to set for 2 hours. The coping screws were unscrewed from the implant replica then the cast was retrieved from the impression. A total of 30 master casts were obtained (10 casts for each group). All the master casts were evaluated 


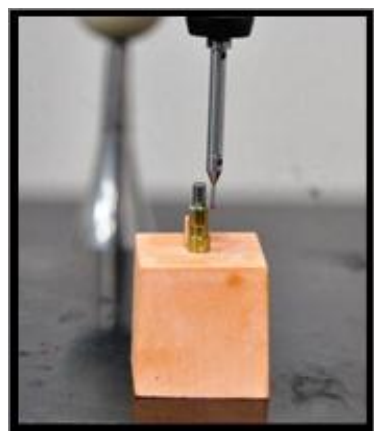

Fig. 5. Measurement of the linear angle on the master cast using a coordinate measuring device.

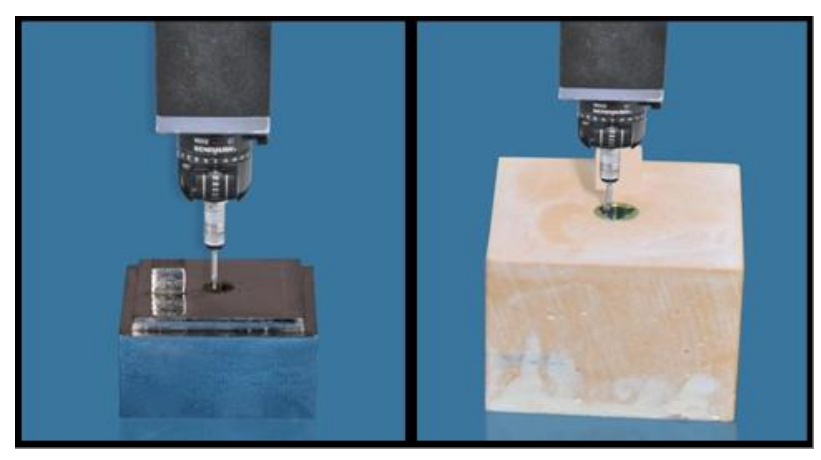

Fig. 6. Measurement of the vertical distance on reference model(left) and master cast (right) using a coordinate measuring device.

using a coordinate measuring machine (CMM, Accurate spectra, Pune, India).

The reference model was measured to obtain the reference values. The open tray impression coping was connected to the implant replica in the reference model. To measure the rotational discrepancy, the linear angle between the lower end of the vertical wall of the rectangular projection and the lower end of the flat surface of the impression coping was measured (Fig. 5). To measure the vertical discrepancy, the distance between the top plane of the rectangular projection and the three reference points on the tri-channel interface of the implant replica was measured. The reference model was measured 5 times to get a mean value. Similarly, all the master casts were measured using the coordinate measuring machine (Fig. 6).

\section{RESULTS}

In Part - I of the study, the peak torque value was re-
Table 1. Comparative evaluation of the rotational resistance offered by vinylpolysiloxane (Group IA), polyether (Group IB), vinylsiloxanether(Group IC) impression materials in implant impression using One Way Analysis of variance (ANOVA)

\begin{tabular}{cccc}
\hline Groups & Mean $(\mathrm{N} \cdot \mathrm{cm})$ & $\begin{array}{l}\text { Standard } \\
\text { deviation }\end{array}$ & P-value \\
\hline Group IA & 9.46 & $>0.01$ & \\
Group IB & 7.94 & $>0.01$ & $0.001^{*}$ \\
Group IC & 3.72 & $>0.01$ & \\
\hline
\end{tabular}

*P<0.05, statistically significant.

Table 2. Comparative evaluation of the vertical distance on the reference model and the master casts prepared from implant impression made with vinylpolysiloxane (Group IIA), polyether (Group IIB) impression materials using One Way Analysis of Variance (ANOVA)

\begin{tabular}{cccc}
\hline Groups & Mean $(\mathrm{mm})$ & $\begin{array}{l}\text { Standard } \\
\text { deviation }\end{array}$ & P-value \\
\hline Reference model & 5.67 & $<0.01$ & \\
Group IIA & 5.64 & $<0.01$ & 0.40 \\
Group IIB & 5.73 & $<0.01$ & \\
\hline
\end{tabular}

$\star P<0.05$, statistically significant.

Table 3. Multiple comparisons of the mean rotational resistance of vinylpolysiloxane (Group IA), polyether (Group IB), vinylsiloxanether (Group IC) impression materials in implant impression using Post-hoc Tukey HSD analysis

\begin{tabular}{cccc}
\hline Groups & Mean $(\mathrm{N} \cdot \mathrm{cm})$ & $\begin{array}{l}\text { Standard } \\
\text { deviation }\end{array}$ & P-value \\
\hline Group IA & 9.46 & $>0.01$ & $<0.01^{*}$ \\
Group IB & 7.94 & $>0.01$ & \\
Group IB & 7.94 & $>0.01$ & $<0.01^{*}$ \\
Group IC & 3.72 & $>0.01$ & \\
Group IC & 3.72 & $>0.01$ & $<0.01^{*}$ \\
Group IA & 9.46 & $>0.01$ & \\
\hline
\end{tabular}

$\star P<0.05$, statistically significant.

corded and represented as the rotational resistance of the impression material. The mean and standard deviation was obtained for each test group and the results were statistically analyzed using one-way ANOVA and Posthoc Tukey HSD test. On comparison of the mean rotational resistance, vinylpolysiloxane (Group IA) showed the highest mean rotational resistance followed by polyether (Group IB), and the least by vinylsiloxanether (Group IC) impression materials $(9.46,7.94$, and $3.72 \mathrm{~N}$ • $\mathrm{cm})$ and was statistically significant. $(\mathrm{P}<0.05)$. On multiple comparisons of the mean rotational resistance within Group IA, Group IB and Group IC using posthoc Tukey 
Table 4. Multiple comparisons of the mean linear angle on the reference model and the master casts prepared fromimplant impression made withvinylpolysiloxane (Group IIA), polyether (Group IIB) impression materials using Post-hoc Tukey HSD analysis

\begin{tabular}{lccc}
\hline \multicolumn{1}{c}{ Groups } & $\begin{array}{c}\text { Mean } \\
\text { (degree) }\end{array}$ & $\begin{array}{c}\text { Standard } \\
\text { deviation }\end{array}$ & P-value \\
\hline Reference model & 0.04 & $<0.01$ & $<0.01 *$ \\
Group IIA & 8.83 & $>0.01$ & \\
Group IIA & 8.83 & $>0.01$ & $<0.01 *$ \\
Group IIB & 4.38 & $>0.01$ & \\
Group IIB & 4.38 & $>0.01$ & $<0.01 *$ \\
Reference model & 0.04 & $<0.01$ & \\
\hline
\end{tabular}

*P<0.05, statistically significant.

HSD showed Group IA had a higher value than Group IB, Group IB had a higher value than Group IC \& Group IA had a higher value than Group IC and was statistically significant $(\mathrm{P}<0.05)$ (Tables 1-4).

In Part - II of the study, The linear angle, and the vertical distance were measured using a coordinate measuring machine for the reference model and all the master casts. The mean and standard deviation was obtained for each test group and the results were statistically analyzed using one-way ANOVA, Post-hoc Tukey HSD test, and Independent ' $t$ ' test. On comparison of the mean linear angle using one way ANOVA test, the reference model showed the least mean linear angle followed by polyether (Group IIB) and the highest by vinylpolysiloxane (Group IIA) $\left(0.04^{\circ}, 8.83^{\circ}\right.$, and $\left.4.38^{\circ}\right)$. The difference was statistically significant $(\mathrm{P}<0.05)$. On multiple comparisons using posthoc, Tukey HSD analysis showed the mean linear angle of Group IIA and Group IIB to be significantly higher than that of the reference model. The mean linear angle of Group IIB was significantly lesser than that for Group IIA, indicating a significantly lesser mean discrepancy between Group IIB \& the reference model than that between Group IIA \& the reference model. In the comparison of the mean linear angle using the independent ' $t$ ' test, vinylsiloxanether (Group IIC) exhibited a statistically significant difference with the reference model $\left(0.04^{\circ}\right.$, $\left.4.9^{\circ}\right)$. The difference was statistically significant $(\mathrm{P}<0.05)$.

Comparison using One Way Analysis of Variance (ANOVA) showed the mean vertical distance of Group IIA and Group IIB to be insignificantly different from that of the reference model, indicating that both Groups IIA
\& IIB had insignificant mean vertical discrepancy when compared to the reference model and also between themselves. On comparison of the mean vertical distance using the independent ' $t$ ' test, vinylsiloxanether (Group IIC) exhibited a statistically insignificant difference with the reference model $(5.63 \mathrm{~mm}, 5.67 \mathrm{~mm})(\mathrm{P}>0.05)$.

\section{DISCUSSION}

An inaccurate impression may result in prosthesis misfits causing mechanical complications include screw loosening, screw fracture, implant fracture, and occlusal inaccuracies ${ }^{2,7,27}$. Biological complications may include adverse tissue reactions, pain, tenderness, marginal bone loss, and loss of osseointegration ${ }^{7,10)}$. The advantage of the closed tray technique is the visual fastening of a replica to the coping and therefore ensuring its complete seating $^{7)}$. However, elastic deformation may result from non-parallel implants while retrieving the impression tray from the mouth ${ }^{7,24)}$.

The open tray technique allows for impression coping to remain within the impression. This reduces the effect of the implant angulation, the deformation of the impression material upon recovery ${ }^{2}$. But during fastening of the analog to the impression coping there are chances of rotation of coping within the impression thereby causing a rotational distortion ${ }^{7,20)}$. Various methods have been suggested by several authors such as splinting, and modification of impression copings to reduce the rotational tendency of the impression coping during the implant replica connection ${ }^{6,11)}$. However, splinting of impression copings is not possible in single implant or randomly distributed single implants in a partially edentulous arch. In such situations, the rotational tendency of the impression coping is influenced by the rigidity of the impression material and the design of the open tray impression coping $^{13)}$. In this study, a reference model with a rectangular projection was fabricated by luting a Nobel Replace implant replica into the cylindrical well of the stainless steel block with Type I glass ionomer cement. This was to resemble the single implant situation in a partially edentulous arch.

Impressions made with vinyl polysiloxane, polyether, and vinylsiloxanether materials have resulted in the rota- 
tional resistance of 9.46, 7.94, and $3.72 \mathrm{~N} \cdot \mathrm{cm}$ respectively. The comparative evaluation of the rotational resistance of three impression materials with Nobel Replace open tray implant level impression coping indicated that vinylpolysiloxane offered the highest rotational resistance followed by polyether and the least by vinylsiloxanether. The difference was statistically significant. The higher mean rotational resistance for the vinylpolysiloxane and polyether materials can be attributed to the favorable shore A hardness number at 1 hour. The difference could be due to the design of the impression coping, the impression materials used, and the testing apparatus used for the evaluation of rotational resistance.

Open tray implant level impressions were made with vinyl polysiloxane, polyether, and vinylsiloxanether impression materials. rotational resistance of the impression materials was evaluated to set the baseline torque value while connecting the implant replica to the impression copings. This baseline $(5 \mathrm{~N} \cdot \mathrm{cm})$ torque value was such that it was below the least value of rotational resistance offered by the vinylpolysiloxane and polyether impression materials. If the baseline torque values exceed the rotational resistance of the impression materials for a particular design of the impression coping, there is a possibility of a complete rotation of the impression coping while connecting to the implant replica. This can result in errors due to the rotational discrepancy mentioned earlier. After the gentle hand tightening of the impression coping to the implant replica, the coping screws were torqued to $5 \mathrm{~N} \cdot \mathrm{cm}$ for vinylpolysiloxane and polyether groups using a digital screw torque checker. Since the lowest rotational resistance value recorded for vinylsiloxanether was $2.8 \mathrm{~N} \cdot \mathrm{cm}$ in the study, a gentle hand tightening was alone given for this group. In few studies evaluating the accuracy of implant impressions, the impression coping was connected to the implant replica by hand tightening ${ }^{3,7,16,28)}$ only, whereas it was torqued to 10 $\mathrm{N} \cdot \mathrm{cm}$ in other studies ${ }^{29)}$. However, the tightening torque achieved with fingers is also subjected to individual variation and can reach up to 11 to $38 \mathrm{~N} \cdot \mathrm{cm}$. There is no consensus reported in the literature for the amount of torque to be given while connecting the implant replica ${ }^{6,11,19)}$.

The mean linear angle of the reference model and mas- ter casts obtained with vinylpolysiloxane, polyether, and vinylsiloxanether materials were $0.04,8.83,4.38,4.9$ degrees respectively. The mean linear angle of the master casts obtained with vinylpolysiloxane, polyether, and vinylsiloxanether material was significantly higher than the reference model. The mean linear angle of the master casts obtained with polyether was significantly lesser than the master casts with vinylpolysiloxane. The lower values of the rotational errors could be attributed to the rigidity and high shore hardness of the polyether impression material in preventing the rotation of the impression coping while applying $5 \mathrm{~N} \cdot \mathrm{cm}$ torque. The higher values for vinylpolysiloxane could be correlated to the lesser shore hardness indicating that the open tray impression coping could be easily rotated within the impression. The results obtained in this present study can be compared with the previous study by Vigolo $^{30}$.

The reports of 1.6 to 5.3-degree tolerance between implant and abutment and the existence of rotational freedom of about 5.5 degrees between implant and abutment suggest that the values obtained in this study were within the clinically acceptable limits for the polyether and vinylsiloxanether groups ${ }^{31)}$. The elasticity of the vinylpolysiloxane could have lead to the rotation of the impression coping while applying $5 \mathrm{~N} \cdot \mathrm{cm}$ torque through its higher rotational resistance. The master casts prepared from vinylsiloxanether exhibited lesser rotational discrepancy than vinylpolysiloxane due to gentle hand tightening through its least rotational resistance.

The mean linear angle of the master casts obtained with vinylsiloxanether was lesser than the vinylpolysiloxane group and marginally higher than the polyether group, though it cannot be statistically compared. However, the studies evaluating the rotational discrepancy with vinylsiloxanether material were lacking in the literature to make direct comparisons of the results obtained in the present study.

The mean vertical distance of the reference model and master casts obtained with vinylpolysiloxane, polyether, and vinylsiloxanether materials were 5.67, 5.64, 5.73, 5.63 $\mathrm{mm}$ respectively. In a comparison of the mean vertical distance, all the master casts prepared from vinylpolysiloxane, polyether, and vinylsiloxanether materials showed no significant difference with the reference 
model. Master casts with vinyl polysiloxane showed the least vertical discrepancy followed by vinylsiloxanether and polyether. The lesser vertical discrepancy associated with vinyl polysiloxane and vinylsiloxanether $(30 \mu \mathrm{m}, 40$ $\mu \mathrm{m})$ could be attributed to its excellent dimensional stability $^{25)}$. The negative values $(60 \mu \mathrm{m})$ for polyether could be correlated to its linear contraction. The mean vertical distance was not influenced by the hand tightening or by the application of $5 \mathrm{~N} \cdot \mathrm{cm}$. All the master casts showed vertical discrepancies in the range of 30 to $60 \mu \mathrm{m}$.

In an open tray implant level impression, the amount of torque to be applied was not mentioned in the literature while connecting the impression coping to the implant and connecting the implant replica to the impression coping after the impression ${ }^{32,33}$. The results obtained in this study clearly showed that the $5 \mathrm{~N} \cdot \mathrm{cm}$ tightening torque of the impression coping has not influenced the vertical relation of the implant/implant replica. To produce the acceptable vertical relation of the implant to abutment/prosthesis, gentle hand tightening of the impression coping to the implant replica will be sufficient. Further torquing of the impression coping may lead to rotation of the impression coping within the open tray impression.

This study had some limitations. we had not explored the influence of saliva, teeth, adjacent structures, the bulk of impression material, the depth of implant placement, and the design of impression copings, also the influence of modification of impression copings like sandblasting, application of tray adhesive, and its combination on rotational resistance and discrepancy have not been considered.

\section{CONCLUSION}

Vinylpolysiloxane showed the highest mean rotational resistance. Polyether showed the least rotational discrepancy. Vinylpolysiloxane showed the least vertical discrepancy.

\section{ORCID}

$$
\begin{aligned}
& \text { Jayakrishnakumar Sampathkumar, } \\
& \text { https:// orcid.org/0000-0003-0047-1999 }
\end{aligned}
$$

\author{
Hariharan Ramakrishnan, \\ https:/ / orcid.org/0000-0003-4466-5744
}

\section{REFERENCES}

1. Misch E. Dental implant prosthetics. 2005, Mosby 3ed, P 17.

2. Carbal M, Guedes G. Comparison analysis of 4 impression techniques for implants. Implant Dent 2007;16:1-9.

3. Kim S, Nicholls JI, Han CH, Lee KW. Displacement of implant components from impressions to definitive casts. Int J Oral Maxillofac Implants 2006;21:747-55.

4. Lee H, So S, Hochstedler L. The accuracy of implant impressions: A systematic review. J Prosthet Dent 2008;100: 285-91.

5. Lee J, Cho B. Accuracy of five implant impression technique effect of splinting materials and methods. J Adv Prosthodont 2011;13:177-85.

6. Assuncao WG, Cardoso A, Gomes EV, Tabata LF. Accuracy of impression techniques for implants. Part 1-Influence of transfer copings surface abrasion. J Prosthodont 2008;17:641-7.

7. Conard J, Pesun J, Delong R, Hodges. Accuracy of two impression techniques with angulated implants. J Prosthet Dent 2007;97:349-56.

8. Vigolo P, Odont Dr, Fonzi F, Majzoub Z, Cordioli G. An evaluation of impression techniques for multiple internal connection implant prostheses. J Prosthet Dent 2004;92:470-6.

9. Amer A, Mostafa H. Evaluation of some factors that may affect the accuracy of implant transfer impression. C D J 2009;2: 219-26.

10. Barbosa S, Bernardes R, Neves das, Neto F, Mattos de, Ribeiro F. Relation between implant/abutment vertical misfit and torque loss of abutment screws. Braz Dent J 2008;19:358-63.

11. Hariharan R, Shankar C, Rajan M, Baig MR, Azhagarasan NS. Evaluation of the accuracy of multiple dental implant impressions using various splinting materials. Int J Oral Maxillofac Implants 2010;25:38-44.

12. Ozkan $\mathrm{Y}$, Ozcan M, Akalin F, Ozkan K. Evaluation of the methods used for impression making for different implant systems in prosthetic dentistry. Brazilian Dental Science 2006;9:21-33.

13. Rashidan N, Alikhasi M, Samadizadeh S, Beyabanaki E. Accuracy of implant impressions with the different impression coping types and shapes. Clin Implant Dent Relat Res 2012;14:218-225.

14. Papaspyridakos P, Chen CJ, Daukoudakis A, Weber HP, Chronopoulos V. Accuracy of implant impressions for partially and completely edentulous patients: A systematic review. Int J Oral Maxillofac Implants 2014;29:836-45.

15. Chee W, Jivraj S. Impression techniques for implant dentistry. Br Dent J 2006;201:429-32.

16. Jo SH, Kim KI, Seo JM. Effect of impression copings and implant angulations on the accuracy of implant impressions: An in vitro study. J Adv Prosthodont 2010;2:128-33

17. Kim JH, Kim KR, Kim S. Critical appraisal of implant impression accuracies: A systematic review. J Prosthet Dent 2015;114: 
185-192.

18. Prithiviraj R, Pujari L, Garg P, Shruthi P. Accuracy of the implant impression obtained from different impression materials and technique: Review. J Clin Exp Dent 2011;3:106-11.

19. Del'Acqua MA,Arioli-Filho JN,Compagnoni MA, Filho JN. Accuracy of impression and pouring techniques for an implant-supported prosthesis. Int J Oral Maxillofac Implants 2008;23:226-36.

20. Faria de, Neves C, Miranda E, Teixeira L. Evaluation of the accuracy of different transfer impression techniques for multiple implants. Braz Oral Res 2011;25:163-7.

21. Kanawati A, Richards MW, Becker JJ. Measurement of clinicians' ability to hand torque dental implant components. J Oral Implantol 2009;35:185-8.

22. Sorrentino R, Gherlone F, Calesini G, Zarone F. Effect of implant angulation, connection length, and impression material on the dimensional accuracy of implant impressions: An in vitro comparative study. Clin Implant Dent Relat Res 2009;6:63-76.

23. Wenz HJ, Hertrampf K. Accuracy of impressions and casts using different implant impression techniques in a multi-implant system with an internal hex connection. Int J Oral Maxillofac Implants 2008; 23:39-47.

24. Craig RG. Restorative dental materials. St. Louis, MO: CV Mosby, 1989: 293-346.

25. Anusavice KJ: Phillip's Science of Dental Materials, 11th ed. St.Louis: Saunders, 2003

26. Vojdani M, Torabi K, Ansarifard E. Accuracy of different im- pression materials in parallel and nonparallel implants. Dent Res J 2015;12: 315-22.

27. Assuncao WG, Cardoso A, Gomes EV, Tabata LF. Accuracy of impression techniques for implants. Part 1-Influence of transfer copings surface abrasion. J Prosthodont 2008;17:641-7.

28. Lee J, Heo J, Koak Y, Kim K. Accuracy of different impression techniques for internal-connection implants. Int J Oral Maxillofac Implants 2009;24:823-30.

29. Hoods-Moonsaamy VJ, Owen P, Howes DG. A comparison of the accuracy of polyether, polyvinylsiloxane, and plaster impressions for a long-span implant-supported prosthesis. Int J Prosthodont 2014;27:433-8.

30. VigoloP, Mutinelli, Fonzi F, Stellini E. An in Vitro evaluation of impression techniques for multiple internal-and external-connection implant prostheses. Int J Oral Maxillofac Implants 2014;29:807-18.

31. Carrilho GPB, Dias RP, Elias CN. Comparison of external and internal hex implants rotational freedom: A pilot study. Int J Prosthodont 2005:18:165-6.

32. Barbosa S, Bernardes R, Neves das, Neto F, Mattos de, Ribeiro F. Relation between implant/abutment vertical misfit and torque loss of abutment screws. Braz Dent J 2008;19:358-63.

33. Filho HG, Mazaro JV, Vedovatto E, Assuncao WG, dos Santos $\mathrm{PH}$. Accuracy of impression techniques for implants. Part 2 Comparison of splinting techniques. J Prosthodont 2009;18: 172-6. 